\title{
Preparation of Nano-Scale Biopolymer Extracted from Coconut Residue and Its Performance as Drag Reducing Agent (DRA)
}

\author{
Muhammad Luqman Bin Hasan ${ }^{1, a}$, Mohamad Naqib Bin Mohamed Khalid ${ }^{1}$ \\ ${ }^{1}$ Department of Petroleum Engineering, Universiti Teknologi PETRONAS, Malaysia
}

\begin{abstract}
Drag or frictional force is defined as force that acts opposite to the object's relative motion through a fluid which then will cause frictional pressure loss in the pipeline. Drag Reducing Agent (DRA) is used to solve this issue and most of the DRAs are synthetic polymers but has some environmental issues. Therefore for this study, biopolymer known as Coconut Residue (CR) is selected as the candidate to replace synthetic polymers DRA. The objective of this study is to evaluate the effectiveness of Nano-scale biopolymer DRA on the application of water injection system. Carboxymethyl cellulose (CMC) is extracted by synthesizing the cellulose extracted from CR under the alkali-catalyzed reaction using monochloroacetic acid. The synthesize process is held in controlled condition whereby the concentration of $\mathrm{NaOH}$ is kept at $60 \% \mathrm{wt}, 60{ }^{\circ} \mathrm{C}$ temperature and the reaction time is 4 hours. For every $25 \mathrm{~g}$ of dried CR used, the mass of synthesized CMC yield is at an average of $23.8 \mathrm{~g}$. The synthesized CMC is then grinded in controlled parameters using the ball milling machine to get the Nano-scale size. The particle size obtained from this is $43.32 \mathrm{Nm}$ which is in range of Nano size. This study proved that Nano-size CMC has higher percentage of drag reduction (\%DR) and flow increase (\%FI) if compared to normal-size CMC when tested in high and low flow rate; $44 \%$ to $48 \%$ increase in $\% \mathrm{DR}$ and $\% \mathrm{FI}$ when tested in low flow rate, and $16 \%$ to $18 \%$ increase in $\% \mathrm{DR}$ and $\% \mathrm{FI}$ when tested in high flow rate. The success of this research shows that Nano-scale DRA can be considered to be used to have better performance in reducing drag.
\end{abstract}

\section{Introduction}

\subsection{Drag}

In oil and gas industry, drag typically occurs in the pathway of fluid inside the injection tubing or pipelines at high flow rate. Zakaria \& Jasamai mentioned that drag, which is also known as friction, is defined as energy that oppose to an object's relative motion through a fluid [1]. In other words, drag will cause a downward force to the fluid and will create drop in pressure of the fluid. Drag are classified as one of the major problems in oil and gas industry as the presence of drag will reduce the flow capacity and subsequently will involve in installation of more pumps as the easiest way to increase the pressure of the fluid in order to overcome the friction pressure losses is by pumping [2], and hence, will contribute in higher operating cost.

As stated by Bailey W. J. \& Peden J. M. (1997), there are two (2) types of flow regime; known as laminar flow and turbulent flow [3]. Drag are not usually occur during laminar flow as the movement of fluid's particles are in parallel or layer manner if compared with turbulent flow, which the fluid's particles are moving in random style, and hence, will have more contact with the pipeline wall to create drag or friction force. However, to show the type of flow regime of fluid in a pipeline, Avila et al. (2011) in their study indicate that flow regime is usually identified by using the dimensionless Reynolds number (NRE) which is defined in equation below [4]:

$$
\mathrm{N}_{\mathrm{Re}}=\frac{\rho v d}{\mu}
$$

Ikoku (1984) said that for flow in a circular pipe, the change from laminar to turbulent flow is typically estimated to happen at N_RE $=2100$ [5]. The fluid behaviors are generally classified as follow:

Table 1. Types of Flow Regime

\begin{tabular}{|c|c|}
\hline $\mathrm{N}_{\mathrm{Re}}<2100$ & Laminar flow \\
\hline $2100<\mathrm{N}_{\mathrm{Re}}<4000$ & Transitional flow \\
\hline $\mathrm{N}_{\mathrm{Re}}>4000$ & Turbulent flow \\
\hline
\end{tabular}

Transitional flow is where laminar flow and turbulent flow occurs at the same time.

\footnotetext{
a luqman.hasan@petronas.com.my
} 


\subsection{Drag Reduction}

Douberet (2009) defined drag reduction as an occurrence where a high cutback (over 70\%) of turbulent friction of a fluid happens due to the inclusion of just a few parts per million of additives [6]. According to Campbell \& Jovancicevic (2001) in their research, the drag reduction can results in increased fluid flow and/or decreased in pressure loss [7]. Therefore, reducing drag in a pipeline is really important to ensure that the flow capacity of a pipeline will be high. Ibrahim \& Braimoh (2005) reported that drag reduction occurs due to suppression of the energy dissipation by turbulent eddy currents near the pipe wall during turbulent flow based from the Drag Reduction Theory [8].

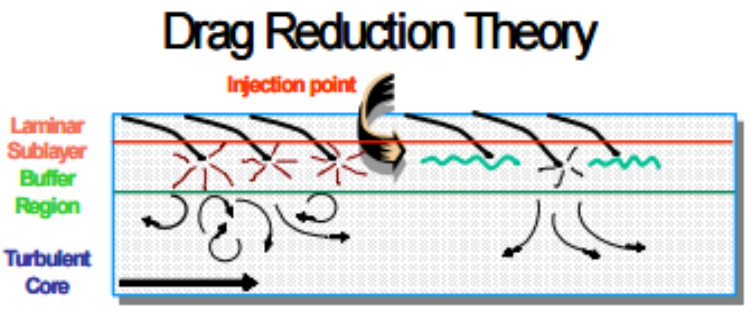

Figure 1. Drag Reduction Theory [8]

From Figure 1, part of laminar sub layer known as "turbulent streak" will move towards the buffer region and starts to oscillate and vortex. It will then tend to move rapidly as it gets closer to the turbulent core and eventually becomes unstable and burst out where it throws fluid into the turbulent core. This phenomenon is known as "turbulent burst" which causes wasted in energy.

Drag reducing agent will disturb the bursting process by dampen the burst and act as shock absorber, thus, reducing the subsequent turbulent bursts. This absorption of burst will result in less wasted energy and subsequently reduces the frictional pressure loss.

In 1906, Blatch conducted a study of "Water Filtrate" and observed that when some addictives were added to a flowing fluid, a significant pressure drop occurred [9]. The theory of his study was then further explained and proved in 1948 by Toms where he observed a decrease in wall friction and pressure drop in a flow with turbulent regime after polymers were added [10]. Tom's study has played an important and major role in introducing the term of drag reduction effect with extensive studies on various source of polymers. The calculation of drag reduction percentage (\%DR) is as follow:

$$
\% D R=\frac{\left|\Delta P_{f}-\Delta P_{f D R A}\right|}{\Delta P_{f}} \times 100
$$

The formula is famously found in most research papers that have been used to check the relationship between the presence of DRA and resulting pressure drop [2].

\subsection{Drag Reducing Agent}

An additive that is made up of high molecular weight polymers which is use to improve the flow of oil in transportation pipeline as well as in water injection system by reducing the pressure drop is called Drag reducing agent, or DRA [9]. Whilst the term drag reduction refers to the decrease of friction in the turbulent flow inside the tubing causing the rise in flow capacity and the decline in pressure loss. Darbouret et. al (2009) stated that the DRA is able to reduce the pressure drop of the fluid if it is categorized as turbulent flow and DRA also helps to boost the tubing capacity [6].

Drag reducing agents which can be classified into three (3) main categories: polymers, surfactants and fibers, are majorly used in the industry due to high efficiency in reducing drag and economical. The polymers are divided into two which are synthetic and natural polymers. Synthetic polymers are made from numerous mixtures of chemicals, while the natural polymer (known as biopolymer) are extracted from natural waste materials. Synthetic polymers are widely used in industry due to their excellence performance in reducing pressure drop and reducing drag but recently there are environmental issues rise as the accumulation of synthetic polymers can seep into the soil and will cause harm to the ecosystem. Based from the issue arises, researchers around the world conducted studies on biopolymers as replacement of synthetic polymers. They conducted many experiments to test on the effectiveness of biopolymers DRA on different parameters of fluid.

The effectiveness of a DRA is depending on several factors, such as the polymer's solubility, molecular weight, cloud point, injection location, salinity of water, flow turbulence, temperature of fluid, degradation, concentration, shear rate etc. As reported by Salamone (1996), the two most important factors that decide the effectiveness of DRA is the linear flexible chain structure and ultra-high-molecular-weight (UHMW) polymers [11]. Percentage of drag reduction (\%DR) can be related with the percent flow increase $(\% \mathrm{FI})$ by using the equation below:

$$
\% F I=\left\{\left(\frac{100}{100-\% D R}\right)^{0.556}-1\right\} \times 100
$$

In order to get the desired concentration, the volume of DRA $\left(\mathrm{V}_{\mathrm{DRA}}\right)$ to be injected into the liquid can be determined by the following equation:

$$
V_{D R A}=\frac{C_{D R A} \times V_{T O T A L}}{1 \times 10^{6}}
$$

The most used polymer as DRA in industries are Carboxymethyl cellulose (CMC), Polyethylene Oxide (PEO), Guar Gum (GGM) and Polyacrylamide (PAM) [20]. Carboxymethyl cellulose (CMC) is the result from the alteration of natural polymer known as cellulose and which is made from the derivation of cellulose resulting 
from the reaction of alkali (e.g. sodium hydroxide) and acid (e.g. monochloroacetic acid). Due to its abundance availability and cheaper price if compared to synthetic polymers, these natural polymers have been used in many studies to test on the best candidates to be the most effective natural polymers DRA as well as to test the effectiveness of natural polymers DRA on different fluid and pipeline parameters. Other than that, these polymers also contain larger molecular weight, which according to Kim (2009) is one of the characteristic of good drag reducers.

After the coconut milk is extracted from the flesh, the wastes produced are call coconut residue. Coconut residues are usually disposed as it cannot be eaten or used again. The reason why coconut is chosen as the DRA candidate is due to the availability of that substance. In 2011, Malaysia ranked the ninth coconut producing country in the world with the estimated total of coconut production of 30,000 MT [15]. It also contains high cellulose which indicates a high quality of DRA. From the studies made by $\mathrm{Ng}$, Tan, Lai, Long, \& Mirhosseini (2010), coconut residues has a total of $72.6 \%$ of cellulose [16].

In this project, Nano size particles will be used. As stated by Filipponi et al. (2012), material that is prepared in Nano particles will have alterations in both physical and chemical properties such as higher shear strength as compared to their bulk materials and more soluble in water since they were having in increased surface-tovolume ratio [12].

\section{Experimental Procedure}

\subsection{Carboxymethyl cellulose (CMC) Extraction and Synthesization}

$\mathrm{CR}$ is washed with tap water and rinsed completely. Then, it is dried in oven (Temperature: $60{ }^{\circ} \mathrm{C}$, Duration: 24 hours). The dried CR is kept in air-tight container.

To eliminate any unwanted products and impurities, $40 \mathrm{~g}$ of $\mathrm{CR}$ is mixed with $1 \mathrm{M}$ of Sodium Hydroxide $(\mathrm{NaOH})$ in a beaker. Then, they are cooked together by using magnetic stirrer hot plate (Temperature: $90{ }^{\circ} \mathrm{C}$, Speed: 200 RPM, Duration: 1 hour). The solution is left to cool down (Duration: 20 - 40 minutes). Then, the mixture is filtered by using sieve and residue is accumulated. The residue is rinsed by using tap water until the dark reddishpurple color turns to light red. To ensure that the $\mathrm{CR}$ is dry enough for the extraction of $\mathrm{CMC}$, it is dried down in oven (Temperature: $60{ }^{\circ} \mathrm{C}$, Duration: 24 hours).

For alkalization, $100 \mathrm{ml}$ with $60 \%$ concentration of $\mathrm{NaOH}$ and $900 \mathrm{ml}$ isopropanol is prepared and mixed together with $25 \mathrm{~g}$ of CR. The mixture is stirred by using magnetic stirrer (Speed: 200 RPM, Duration: 30 minutes). To initiate carboxylmethylation reaction, $36 \mathrm{~g}$ of monochloroacetic acid is added into the solution. The solution is stirred until the mixture becomes clear. (Speed: 200 RPM, Duration: 30 minutes, Temperature: $60{ }^{\circ} \mathrm{C}$ ). The temperature of the mixture is kept constant at $60{ }^{\circ} \mathrm{C}$ by gradually checking the thermometer. After 30 minutes of stirring, the polymers started to become thicker and the magnetic stirrer could not be able to stir it. The beaker is then transferred into oven. (Duration: 210 minutes, Temperature: $60{ }^{\circ} \mathrm{C}$ ). Polymer is separated from the mixture by using sieve. It is then suspended into a beaker with $100 \mathrm{ml}$ of methanol with $70 \% \mathrm{v} / \mathrm{v}$ concentration (Duration: 24 hours).

$100 \mathrm{ml}$ glacial acetic acid is poured into the beaker for neutralization and left for a while (Duration: 15 minutes). Then, the neutralized polymer is filtered from the solution by using sieve. To ensure any unwanted by products are removed, the polymer is suspended in 300 $\mathrm{ml}$ of ethanol with $70 \% \mathrm{v} / \mathrm{v}$ concentration (Duration: 10 minutes). Then, the polymer is separated by using sieve. It is then washed by using $300 \mathrm{ml}$ of absolute methanol. The washing activity by using methanol is repeated for 3 times. Then, the polymer is separated by using sieve and then it is dried in the oven (Temperature: $60{ }^{\circ} \mathrm{C}$, Duration: 24 hours). After 24 hours of drying, the extracted CMC sample is grinded by using mortar grinder until become powder size. CMC sample is stored in air-tight container.

\subsection{Planetary Mill (Nano-scale Grinding)}

Table 2. Planetary Mill Test Parameters Setup

\begin{tabular}{|c|c|}
\hline Parameters & Description \\
\hline Rotation Speed & $330 \mathrm{rpm}$ \\
\hline Ball Weight : Powder Ratio & $10: 1$ \\
\hline Grinding Time for 1 Cycle & $\begin{array}{c}6 \text { minutes operation and 4 } \\
\text { minutes stop }\end{array}$ \\
\hline Total Grinding Time Needed & $\begin{array}{c}42 \text { hours }=252 \text { cycle }(7 \\
\text { hours/day for } 6 \text { days })\end{array}$ \\
\hline
\end{tabular}

Table 3. Grinding Ball Calculation

\begin{tabular}{|c|c|c|}
\hline Diameter & Ball needed & Weight \\
\hline $3 \mathrm{~cm}$ & $1 \times 74 \mathrm{~g}$ & $74 \mathrm{~g}$ \\
\hline $2 \mathrm{~cm}$ & $1 \times 23 \mathrm{~g}$ & $23 \mathrm{~g}$ \\
\hline $1 \mathrm{~cm}$ & $20 \times 2.4 \mathrm{~g}$ & $48 \mathrm{~g}$ \\
\hline $0.5 \mathrm{~mm}$ & $\pm 300 \times \pm 0.05 \mathrm{~g}$ & $15 \mathrm{~g}$ \\
\hline \multicolumn{3}{|c|}{ Total Weight } \\
\hline
\end{tabular}

This process involves 2 grinding jars. $16 \mathrm{~g}$ of CMC powder and $160 \mathrm{~g}$ of grinding balls are placed into each grinding jar. The hood is opened and the jars are placed at designated arms. The parameters on the machine is keyed in which includes the rotational speed, time per cycle and total cycle as stated in Table 2. The hood will be automatically locked upon the running of the machine. The main power of the machine is turned off after the grinding process is over. 


\subsection{Fluid Friction Apparatus Test}

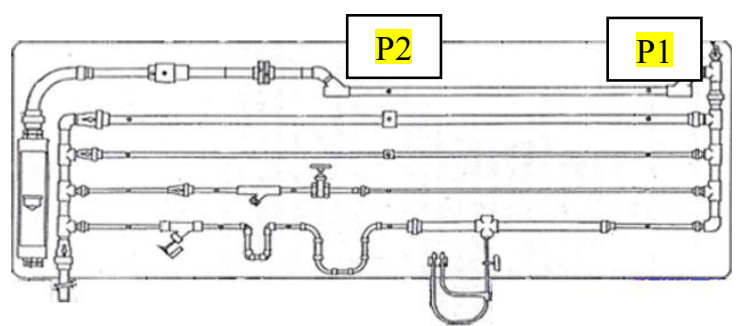

Figure 2. Schematic Diagram of Fluid Friction Apparatus

Flow loop tank is filled with 40 liters of tap water. The pump is switched on and the choke valve is opened until the flowmeter reading is stabilized at rate of $1 \mathrm{~m} 3 / \mathrm{h}$. The flow loop is left circulated for 15 minutes to ensure no air inside the pipe. The water manometer readings which are labeled as point P1 and P2 in Figure 2 are observed and recorded. The experiment is continued by slowly increase the liquid flow rate to $2 \mathrm{~m}^{3} / \mathrm{h}$ by adjusting the flow control valve and monitoring the flowmeter. Again, the water manometer readings are observed and recorded after it is stabilized for 1 minute. The pump is then switched off and the drain valve at the side of the tank is fully opened to drain all water from the tank to prepare for the new run and then flushed once with clean water to remove any leftovers inside the system. The experiments are repeated for Nano-scale (Sample $\mathrm{C} 1$ and $\mathrm{C} 2$ ) and normal-scale DRA solution (Sample C1 and C2). Results are recorded and tabulated.

\section{Result and Discussion}

\subsection{CMC Extraction and Synthesization}

Table 4. Mass of CMC Obtained After Synthesization

\begin{tabular}{|c|c|c|}
\hline \multirow{2}{*}{ Batch No. } & Materials & Yield \\
\cline { 2 - 3 } & CR Cellulose $(\mathrm{g})$ & CMC $(\mathrm{g})$ \\
\hline 1 & 25 & 23.61 \\
\hline 2 & 25 & 23.84 \\
\hline 3 & 25 & 23.96 \\
\hline \multicolumn{2}{|c|}{ TOTAL } & $\mathbf{7 1 . 4 1}$ \\
\hline
\end{tabular}

Three batches of CMC sample have been prepared and the results are tabulated in the Table 4 . Table 4 shows an average of $23.8 \mathrm{~g}$ of CMC powder are produced for every $25 \mathrm{~g}$ of CR cellulose used. The $4.79 \%$ loss of mass may occurred during the filtration process and transportation of the CMC. Despite of the losses, the synthesization has managed to achieve a total of $71.41 \mathrm{~g}$ of CMC powder which will then be used for the next procedure.

\subsection{Preparation of Nano Size CMC}

After grinding the CMC powder by using the Ball Milling Machine, the powder obtained is then tested using Particle Size Analyzer to determine whether the size reaches the Nano size $(<100 \mathrm{~nm})$ or not.

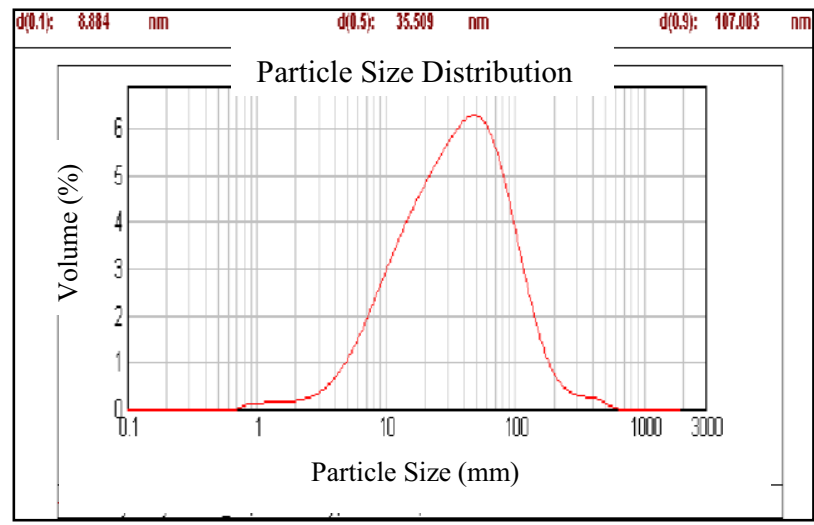

Figure 3. Size Distribution Graph (C1)

Figure 3 shows the size distribution of sample $\mathrm{C} 1$. $10 \%$ of the sample has an average size of $8.884 \mathrm{Nm}$, while $50 \%$ of the sample has an average size of 35.509 $\mathrm{Nm}, 90 \%$ of the sample has an average size of 107.003 $\mathrm{Nm}$. The smallest particle size is around $0.724 \mathrm{Nm}$, and the largest is $549.541 \mathrm{Nm}$. The average size of sample C1 particles is $47.541 \mathrm{Nm}$.



Figure 4. Size Distribution Graph (C2)

Figure 4 shows the size distribution of sample C2. $10 \%$ of the sample has an average size of $3.394 \mathrm{Nm}$, while $50 \%$ of the sample has an average size of 8.369 $\mathrm{Nm}, 90 \%$ of the sample has an average size of 170.840 $\mathrm{Nm}$. The smallest particle size is around $1.259 \mathrm{Nm}$ and the largest is $416.869 \mathrm{Nm}$. The average size of sample C2 particles is $39.098 \mathrm{Nm}$.

From the result of the Size Particle Size test, it shows that both of the samples have the average size of $43.32 \mathrm{~nm}$, thus proving that Nano-scale has been achieved from the ball milling of CMC.

\subsection{Reynolds Number}

DRA can only work effectively in a turbulent regime flow where the Reynolds number is greater than 4000 $(\mathrm{NRe}>4000)$. To confirm the flow regime, the Reynolds number of the flow in the pipeline is calculated by using Equation 1. 
The parameters used to calculate Reynolds number are as follow:

Table 5. Parameters to Calculate Reynolds Number

\begin{tabular}{|c|c|}
\hline Density of solution $\left(\mathrm{kg} / \mathrm{m}^{3}\right)$ & $\mathbf{1 0 1 7 . 5}$ \\
\hline Flow rate $\left(\mathrm{m}^{3} / \mathrm{s}\right)$ & $\mathbf{2 . 7 7 8} \times \mathbf{1 0}^{-4}$ \\
\hline Diameter $(\mathrm{m})$ & $\mathbf{0 . 0 2 3 5}$ \\
\hline Viscosity of solution $(\mathrm{kg} / \mathrm{m} . \mathrm{s})$ & $\mathbf{1 . 0 9} \times \mathbf{1 0}^{-\mathbf{3}}$ \\
\hline Velocity $(\mathrm{m} / \mathrm{s})$ & $\mathbf{0 . 6 4 0 4 8}$ \\
\hline
\end{tabular}

The Reynolds number is as follow:

$$
\begin{gathered}
N_{\mathrm{Re}}=\frac{1017.5 \times 0.64048 \times 0.0235}{1.09 \times 10^{-3}} \\
N_{\mathrm{Re}}=14050.16
\end{gathered}
$$

Since $\mathbf{N}_{\mathbf{R e}}>\mathbf{4 0 0 0}$, thus the flow regime is turbulent.

\subsection{Results from Fluid Friction Test}

The fluid friction test uses manometer readings that give the value of water pressure at the entry of the pipe (P2) and the water pressure at 1 meter from the entry (P1) in the unit of centimeter $(\mathrm{cm})$. Few assumptions have been made for the fluid friction test which are:

i. The solution prepared is homogeneous and the CMC powder has been mixed thoroughly with the water.

ii. The pipe used for the test is assumed to have smooth surface, thus, the effect of friction in the experiment is neglected.

The values of pressure that in centimeter are converted into psi unit by using the hydrostatic pressure formula as below:

$$
\Delta P=(\rho g \Delta h) \times\left(1.45038 \times 10^{-4}\right)
$$

The difference between the value of $\mathrm{P} 2$ and $\mathrm{P} 1$ represents the pressure drop inside the pipe. Table 6 below shows the value of pressure drop from the fluid friction test when there is no CMC (tap water) added:

Table 6. Pressure Drop Using Tap Water (Baseline)

\begin{tabular}{|c|c|c|c|c|}
\hline \multirow{2}{*}{$\begin{array}{c}\text { Flow rate } \\
\left(\mathbf{m}^{\mathbf{3}} / \mathbf{h}\right)\end{array}$} & \multicolumn{4}{|c|}{ Tap Water } \\
\cline { 2 - 5 } & $\mathbf{P 2} \mathbf{( c m )}$ & $\mathbf{P 1} \mathbf{( c m )}$ & $\mathbf{\Delta P}(\mathbf{c m})$ & $\Delta \mathbf{P}(\mathbf{p s i})$ \\
\hline $\mathbf{1}$ & 53.1 & 46.9 & 6.2 & 0.088215 \\
\hline $\mathbf{2}$ & 55.7 & 44.3 & 11.4 & 0.162201 \\
\hline
\end{tabular}

The value of the pressure drop when using tap water only will be used to calculate the drag reduction percentage $(\% \mathrm{DR})$ which is calculated by using Equation 2 . Then, by using the $\% \mathrm{DR}$ value, the flow increase percentage $(\% \mathrm{FI})$ can be calculated by using Equation 3 .

The graph in Figure 5 shows a significant increase of drag reduction percentage. It can be seen that the percentage of flow increased as the flow rate increases.



Figure 5. Summary Result for Fluid Friction Test

For the normal size biopolymer DRA, the increment is almost $51 \%$ increase in $\% \mathrm{DR}$ and $56 \%$ increase in $\% \mathrm{FI}$ when the flow rate is increased from $1 \mathrm{~m}^{3} / \mathrm{h}$ to $2 \mathrm{~m}^{3} / \mathrm{h}$. For Nano size biopolymer, the \%DR and \%FI increases $21 \%$ and $24 \%$ respectively when the flow rate increases. This is due to the high friction force inside the pipeline that caused by the high flow rate that will lead to a high pressure drop. Therefore, CMC's effect in increasing the pressure drop is more visible in the case of higher flow rate compared with lower flow rate.

It can also be observed that the value of $\% \mathrm{DR}$ and $\% \mathrm{FI}$ is much higher when using Nano-scale CMC compared to normal scale CMC. In $1 \mathrm{~m}^{3} / \mathrm{h}$ flow rate, normal scale CMC gives, \%DR reading of $7.258 \%$, and the $\% \mathrm{FI}$ is $4.285 \%$. The value of $\% \mathrm{DR}$ and $\% \mathrm{FI}$ increases to $10.484 \%$ and $6.355 \%$ respectively when using Nano size CMC. This shows an increment of $44 \%$ for $\% \mathrm{DR}$ and increment of $48 \%$ for $\%$ FI. In $2 \mathrm{~m}^{3} / \mathrm{h}$ flow rate case, the $\% \mathrm{DR}$ is $10.965 \%$, and the $\% \mathrm{FI}$ is $6.672 \%$ when using normal scale CMC. When using Nano size CMC, the value of $\% \mathrm{DR}$ and $\% \mathrm{FI}$ increases to $12.719 \%$ and $7.858 \%$ respectively. This shows an increment of $16 \%$ for $\% \mathrm{DR}$ and increment of $18 \%$ for $\% \mathrm{FI}$.

The higher percentage of drag reduction and flow increase in Nano-scale solution compared to normal-scale solution proves that the Nano-scale CMC has better ability to reduce drag. This is due to two reasons; the higher solubility and the better alterations in physical and chemical properties. Since the Nano size materials have larger surface area, it will allow the materials to be dissolved in solution much better and thus can increase the efficiency to decrease drag. Normal size materials will have difficulties to dissolve completely in the solution. The assumption that the powder has dissolved completely is only made by observation, whereby due to the solution is homogenous, it is assumed that the powder have completely dissolved. However realistically, there might be particles that are not fully dissolved when using the normal size materials. This will then affect the performance of the CMC to reduce drag.

Nano materials also have better ability to react with water in reducing drag. The bursting process created from turbulence flow can be effectively dampen by the Nano particles due to the large surface area of the materials. 


\section{Conclusion}

Friction force or drag does exist in a turbulent flow of fluid in a pipeline. This issue is really critical for oil and gas industry especially during the transportation of oil and re-injection of water during water flooding, as it can cause drop in pressure and reduction of flow capacity. From previous researches that have been done, pressure of the fluid can be increased by using pumps, but, it will involve higher cost. So, drag reduction technique has been used to tackle the problem. Drag reduction is where the pressure loss is decreased and the flow performance will be increased by just adding additives called Drag Reducing Agents (DRA). This study shows that a biopolymer DRA can be extracted from coconut residue (CR) which is more environmental friendly.

The study shows that for every $25 \mathrm{~g}$ of dried CR used, it can yield an average of $23.8 \mathrm{~g}$ of synthesized CMC under controlled conditions. The CMC yield is grinded into Nano-scale particle using ball milling machine using controlled parameters. The process yields CMC powder with an average particles size of $43.32 \mathrm{Nm}$, which is in range of Nano particle size $(<100 \mathrm{Nm})$. Both of Nano scale and normal scale CMC are tested by using the fluid friction apparatus to test on the ability to reduce pressure drop. The experiment shows that Nano-size $\mathrm{CMC}$ has higher percentage of drag reduction $(\% \mathrm{DR})$ and flow increase $(\% \mathrm{FI})$ if compare with normal-size CMC when tested in high and low flow rate; $44 \%$ to $48 \%$ increase in $\% \mathrm{DR}$ and $\% \mathrm{FI}$ when tested in low flow rate, and $16 \%$ to $18 \%$ increase in $\% \mathrm{DR}$ and $\% \mathrm{FI}$ when tested in high flow rate. The success of this research shows that the $\mathrm{CMC}$ extracted from coconut residue can have the potential to be used to replace the current synthetic DRAs and also Nano-scale DRA can be considered to be used to have better performance in reducing drag.

$\mathrm{N}_{\mathrm{Re}}=$ Reynolds Number

\section{NOMENCLATURE}

$\% \mathrm{DR}=$ Drag Reduction Percentage

$\Delta \mathrm{P}_{\mathrm{f}}=$ Pressure Drop before injecting DRA

$\Delta \mathrm{P}_{\text {fdra }}=$ Pressure Drop after injecting DRA

$\% \mathrm{FI}=$ Flow Increase Percentage

$\mathrm{V}_{\text {DRA }}=$ Volume of DRA to be injected

$\mathrm{C}_{\mathrm{DRA}}=$ Concentration of DRA solution

$\mathrm{V}_{\text {TOTAL }}=$ Total volume solution

$\mathrm{CMC}=$ Carboxymethylcellulose

$\mathrm{CR}=$ Coconut Residue

DRA $=$ Drag Reducing Agent

$\mathrm{NaOH}=$ Sodium Hydroxide

$\mathrm{Wt} \%=$ Concentration solution on percentage by weight $\mathrm{v} / \mathrm{v}=$ volume to volume concentration

\section{References}

[1] Zakaria, A. N. and Jasamai, M., "To Evaluate Effectiveness of Hibiscus leaves as Drag Reducing Agent in Water System," Universiti Teknologi PETRONAS, Malaysia.
[2] Karami, H. R. and Mowla, D., "Investigation of the effect of various parameters on pressure drop reduction in crude oil pipelines by drag reducing agents," Journal of Non-Newtonian Fluid Mechanics, pp. 37-45, 2012.

[3] Bailey, W. J. and Peden, J. M. "A Generalised and Consistent Pressure Drop and Flow Regime Transition Model for Drilling Hydraulics Suitable for Slimhole, Underbalanced and Horizontal Well." SPE 39281(paper presented at the SPE/IADC Middle East Drilling Technology Conference held in Bahrain, 23-25 November 1997)

[4] Avila, K., Moxey, D., de Lozar, A., Avila, M., Barkley, D., \& Hof, B. (2011). The onset of turbulence in pipe flow. Science, 333(6039), 192-196.

[5] Ikoku, C. U. (1984). Natural gas production engineering: Wiley.

[6] Darbouret, M., Henaut, I., Palermo, T., Glenat, P., and Hurtevent, C., "Experimental Methodology To Evaluate DRAs: Effect of Water Content and Waxes on Their Efficiency," presented at the SPE International Symposium on Oilfield Chemistry, The Woodlands. Texas, 2009.

[7] Campbell, S. E., \& Jovancicevic, V. (2001). Performance Improvements From Chemical Drag Reducers.

[8] Ibrahim, J. U. and Braimoh, L. A., "Drag Reducing Agent Test Result for Chevron Texaco, Eastern Operations, Nigeria (SPE 98819)," in Society of Petroleum Engineers, Abuja, Nigeria, 2005.

[9] Blatch, N. S., "Water Filtrate," ASCE, pp. 400-408, 1906.

[10] Toms, B. A., "Some observation on the flow of linear polymer solution through straight tubes at large Reynolds number," in Proceedings of the 1st International Congress on Rheology, North Holland, Amsterdam, 1948, pp. 135-141.

[11] Salamone, J. C. (1996). Polymeric Materials Encyclopedia, Twelve Volume Set: Taylor \& Francis.

[12] Filipponi, L. and Sutherland, D. (2012). NANOTECHNOLOGIES: Principles, Applications, Implications and Hands-on Activities. Luxembourg: Publications Office of the European Union.

[14] Choi, H., Moin, P. and Kim, J., 1992, “Turbulent drag reduction: Studies of feedback control and flow over riblets," Technical Report TF-55, Stanford University

[15] United Nations Conference on Trade and Development (2011), World Coconut Oil Production. Retrieved on $18^{\text {th }}$ June 2015 from http://www.unctad.info/en/Infocomm/AACPProducts/COMMODITY-PROFILE---Coconut 2/

[16] Ng, S., Tan, C. P., Lai, O. M., Long, K., \& Mirhosseini, H. (2010). Extraction and characterization of dietary fiber from coconut residue. J Food Agric Environ, 8, 172-177 